EPJ Web of Conferences 38, 16001 (2012)

DOI: $10.1051 /$ epjconf/20123816001

(C) Owned by the authors, published by EDP Sciences, 2012

\title{
Extreme quadrupole deformation and clusterization
}

\author{
J. Darai ${ }^{1, a}$, J. Cseh $^{2}$, G. Adamian ${ }^{3}$, and N. Antonenko ${ }^{3}$ \\ 1 Department of Experimental Physics, University of Debrecen, Debrecen, Pf. 105, Hungary-4010 \\ 2 Institute of Nuclear Research, Hungarian Academy of Sciences, Debrecen, Pf. 51, Hungary-4001 \\ 3 Bogoliubov Laboratory, Joint Institute for Nuclear Research, 141980 Dubna, Russia
}

\begin{abstract}
We discuss a simple symmetry-adapted method for the determination of the shape isomers, and for the study of their possible fragmentation. In other words the connection between the quadrupole (collective) and dipole (cluster) degrees of freedom is considered in terms of an easily applicable, yet microscopic method. The energetics is taken into account by the double-folding method. Special attention is focused on those cases in which the theoretical predictions have a direct comparison with experimental observation.
\end{abstract}

\section{Introduction}

The behavior of the finite nuclear matter under extreme conditions raises many exciting questions. One of them concerns the case of the large quadrupole deformation. Superdeformed (SD) states have been observed in several nuclei, while the appearance of hyperdeformation (HD) is a hot topic for a discussion. Especially interesting is their existence in the $N=Z$ nuclei, in which the role of pairing, quartering, isospin, can be studied. The experimental observation of the SD states in these nuclei started approximately a decade.

The investigation of clusterization of a specific nuclear state is important from two aspects. First it contributes to our understanding of the nuclear structure. Second it gives valuable information on the nuclear reactions, which can populate the state in question. In particular, a binary cluster configuration with the clusters in their ground intrinsic state is uniquely related to a reaction channel, like target and projectile in their ground state. Actually, one of the possible definition of a binary cluster configuration is provided by this kind of reaction channel. (Since it is directly related to its observation, probably this is the best way to define a cluster configuration.)

In this contribution we present a simple method, that we have applied recently for the determination of the shape isomers of some light nuclei, and for investigating their possible binary clusterizations as well. In both steps symmetry-considerations played an essential role.

The next section describes briefly the methodology, while in Sec. 3 we present some results. We focus on those cases in which the theoretical predictions or results can be compared with the experimental observation.

\section{Methods}

\subsection{Preliminaries}

The basic connection between the quadrupole shape and clusterization was found in the fifties, via the bridge of

\footnotetext{
a e-mail: darai@atomki.mta.hu
}

the shell model. This relation is based on the $S U(3)$ symmetry. Elliot has shown [1], how the quadrupole deformation and collective rotation can be derived from the spherical shell model: the states belonging to a collective band are determined by their specific $S U(3)$ symmetry. Wildermuth and Kanellopoulos [2] established the relation between the shell and cluster models. They have shown that the Hamiltonians of the two models can be rewritten into each other exactly in the harmonic oscillator approximation. This relation results in a close connection between the corresponding eigenvectors, too: the wave function of one model is a linear combination of those of the other, which belong to the same energy. Later on this relation was interpreted by Bayman and Bohr [3] in terms of the $S U(3)$ symmetry. As a consequence, the cluster bands can be considered from the shell model viewpoint, like a set of states having special $S U(3)$ symmetry, just like the collective rotational bands.

The $1958 S U(3)$ connection was based on the symmetry of the spherical shell model. In light of the fact that today very largely deformed states are known experimentally, it is an important question, what happens to this symmetry with increasing deformation. The superdeformed states e.g. represent a situation which is close to the axially symmetric spheroid with ratios of main axis of $2: 1: 1$, the hyperdeformed states correspond to $3: 1: 1$.

In [4] it was shown that the symmetry algebra of the anisotropic harmonic oscillator is $S U(3)$, when its frequencies are commensurate, i.e. expressed as ratios of integer numbers. As a special case it includes the spherical oscillator, as well as the superdeformed or hyperdeformed shapes. More details, concerning the axially symmetric case with $2: 1,3: 1$, and $3: 2$ ratios are discussed in [5], and for the triaxially deformed oscillator in [6]. (For previous works on this problem we refer to the citations in these papers.)

The connection between the anisotropic harmonic oscillator and clusterization have been discussed in [7-9].

\subsection{Shape isomers}

In what follows we pay attention to the problem of more realistic interactions. In these considerations the Nilsson

This is an Open Access article distributed under the terms of the Creative Commons Attribution License 2.0, which permits unrestricted use, distribution, and reproduction in any medium, provided the original work is properly cited. 
model plays an important role, which gives single nucleon orbitals for more realistic interactions, including spin-orbit, as well as $l^{2}$ terms. Soon after the experimental discovery of the superdeformed states, Sugawara-Tanabe et al. have realized that the $L-S$ coupling recovers for the superdeformed shape, first in a simple model [10], then in more realistic calculations [11]. In particular they found that the $L-S$ coupled spherical wave function components became more that $85 \%$ of the total. The reason for this is the dominant role of the quadrupole interaction due to the large deformation. This phenomenon provides an explanation for the appearance of the parity doublet levels.

We investigate the survival (or appearance) of the $S U(3)$ symmetry systematically as a function of the quadrupole deformation (including triaxial deformation). We do so in terms of the Nilsson-model combined with the concept of the quasi-dynamical (or effective) $U(3)$ symmetry [12]. It is a generalization of the concept of the real $U(3)$ symmetry, known to be approximately valid in light nuclei [1]. The quasi-dynamical symmetry is more general in the following sense. The Hamiltonian breaks the symmetry in such a way that the $U(3)$ quantum numbers are not valid for its eigenvectors either (contrary to the case of the real $U(3)$ dynamical symmetry). In other words neither the operator is symmetric (i.e. it is not a $U(3)$ scalar), nor its eigenvectors are (i.e. they do not transform according to a single irreducible representation) [13]. Yet, the symmetry is present is some sense.

An asymptotic Nilsson-state serves as an intrinsic state for the quasi-dynamical $S U(3)$ representation. Thus the effective quantum numbers are determined by the Nilssonstates in the regime of large deformation [14]. When the deformation is not large enough, then we can expand the Nilsson-states in the asymptotic basis, and calculate the effective quantum numbers based on this expansion [15].

The $S U(3)$ quantum numbers uniquely determine the quadrupole shape of the nucleus [16], thus we obtain the shape isomers from them. In particular, a self-consistency calculation is performed with respect to the quadrupole shape of nucleus, which is an alternative of the more usual energy-minimum calculations. It is based on the application of the quasi-dynamical $U(3)$ quantum numbers [17], and in those cases when a detailed comparison can be made with the traditional energy-minimum calculations, the results are very similar [17-19].

\subsection{Clusterization}

Once the shape isomers have been found, the next question is how they are related to cluster configurations. In simple cases the $S U(3)$ connection could provide the answer [20]. It was working very simple (e.g. in the ground-state region) due to two facts that: i) the $S U(3)$ symmetry was approximately valid, and ii) the connection between the cluster and shell model states was simple, i.e. expanding the cluster basis state in terms of shell model basis only a very few (sometimes a single) basis states gave contribution [21, 22]. The symmetry-breaking interaction, however, mixes the $S U(3)$ states. Furthermore, the shell-model expansion of a cluster state is usually very complicated, too; i.e. many shell-model basis states give contribution [21,22].

What happens with the shape isomers? As seen above, the $S U(3)$ symmetry recovers, even for the symmetry-brea- king interactions like spin-orbit. It is not necessarily a real $S U(3)$, more often it is a quasi-dynamical $S U(3)$, but for light nuclei many times the two coincide with each other. What can we expect from a selection rule, based on the quasi-dynamical $S U(3)$ symmetry? First it has a definite geometrical content in spite of the abstract algebraic formulation. It shows how much the quadrupole shapes of the shell-model state and the cluster configuration are consistent with each other [18]. This consistency is obtained from a cluster picture, in which the Pauli-principle is taken into account at an approximate level. Namely, single shellmodel configurations can be associated to the internal structure of the clusters [14], and for these configurations the exclusion principle is valid. Of course, when the quasidynamical symmetry reduces to a real one, the selection rule gives an exact result (to the extent the $U(3)$ symmetry is valid). Thus we can say that the Pauli-principle is incorporated only in an approximate, but well-defined way, and its validity can be checked by making a comparison with the results of the fully microscopic description, where they are available.

The selection rule is only a necessary condition for the appearance of the allowed clusterization, but not a sufficient one. Another simple prescription is provided by Harvey [23] which is also based on the use of the harmonic oscillator basis, therefore, it is easily applicable, too. This is also a necessary condition. The content of the two requirement is not the same, in some sense, they are complementary to each other. Their relation is discussed more in detail in [19]. Therefore, one should apply both prescription in a combined way, and that is how we used them in the cases presented later on. When a cluster configuration is forbidden, we characterize its forbiddenness in a quantitative way [24].

In short this part can be summarized, by saying that condition i), i.e. the validity of the $U(3)$ symmetry is satisfied (in an approximate way). It also turns out that condition ii), i.e. the simple relation between the cluster and shell model basis is also valid many times, in spite of the fact that the shape isomers are usually highly excited states. It is a consequence of the fact that the highly deformed states are realized only in a very limited number of shellconfigurations.

The energetic preference represents a complementary viewpoint for the selection of clusterization. We incorporate it in two different ways: i) by applying simple bindingenergy arguments [25], and ii) by performing double-folding calculations, according to the dinuclear system model $[26,27]$. In this model the charge (mass) asymmetry $\eta_{Z}=$ $\left(Z_{1}-Z_{2}\right) /\left(Z_{1}+Z_{2}\right)\left(\eta=\left(A_{1}-A_{2}\right) /\left(A_{1}+A_{2}\right)\right)$, where $Z_{1}\left(A_{1}\right)$ and $Z_{2}\left(A_{2}\right)$ are the charge (mass) numbers of the heavy and light nuclei of the dinuclear system, is the relevant collective variable describing the partition of nucleons among the nuclei forming the dinuclear system. The wave function in $\eta_{Z}$ is thought to be a superposition of the mononucleus configuration with $\left|\eta_{Z}\right|=1$ and different cluster-type configurations. The relative contribution of each cluster component to the total wave function is ruled by the potential $U\left(\eta_{Z}\right)$ at $\left|\eta_{Z}\right|<1[27,28]$ :

$$
U\left(\eta_{Z}\right)=V\left(R=R_{m}, \eta_{Z}\right)+B_{1}\left(\eta_{Z}\right)+B_{2}\left(\eta_{Z}\right)_{B}
$$

In the case of $Z=N$ nuclei, $\eta_{Z}=\eta$. The internuclear distance of $R_{m}=R_{t}+0.5 \mathrm{fm}$ corresponds to the minimum 
of the nucleus-nucleus potential $V$. Here $R_{t}$ is the touching distance between the clusters, which depends on their relative orientation. The negative quantities $B_{1}$ and $B_{2}$ are the binding energies of the clusters and $B$ is the binding energy of the parent nucleus. The experimental groundstate masses and quadrupole deformation parameters [29, $30]$ are used in the present calculations. Since the values of $U$ are given with respect to $\mathrm{B}, U\left(\left|\eta_{Z}\right|=1\right)=0$. The double-folding method of the calculation of $V$ as well as the parameters used are presented in $[28,31]$. The mutual orientation of nuclei in the dinuclear system corresponds approximately to the result of the microscopic consideration.

\section{Results}

\section{$3.1^{28} \mathrm{Si}$}

There has been much discussion recently on the superdeformed state in ${ }^{28} \mathrm{Si}$, both from the theoretical and from the experimental sides. An AMD study by Taniguchi et al. [32] delineates an SD band in ${ }^{28} \mathrm{Si}$ with a moment of inertia of $\approx 6 \hbar^{2} / \mathrm{MeV}$. Its structure is dominated by ${ }^{24} \mathrm{Mg}+\alpha$ and ${ }^{12} \mathrm{C}+{ }^{16} \mathrm{O}$ clustering. Another recent calculation in terms of the macroscopic-microscopic model [33] also predicts an SD band with strong ${ }^{12} \mathrm{C}+{ }^{16} \mathrm{O}$ clustering.

From the experimental side, there are a set of states in ${ }^{28} \mathrm{Si}$ identified in the ${ }^{12} \mathrm{C}\left({ }^{20} \mathrm{Ne}, \alpha\right){ }^{28} \mathrm{Si}$ reaction which have been attributed to the superdeformed band by Kubono et al. [34]. This sequence has no, however, the smooth characteristics expected for such a band.

Jenkins et al. have reviewed the available experimental data, and extended them with new $\gamma$-transitions [35]. As a result, they propose a new candidate for the SD band. In particular the $6^{+}$state was identified by Brenneisen et al. [36] at $12.86 \mathrm{MeV}$, which is populated in $(\alpha, \gamma)$ reaction, but not in $(p, \gamma)$. In a recent Gammasphere measurement the ${ }^{12} \mathrm{C}\left({ }^{20} \mathrm{Ne}, \alpha\right){ }^{28} \mathrm{Si}$ reaction was studied [35]. Double and triple $\gamma$-coincidence were measured, and nearly all states below $10 \mathrm{MeV}$ have been located, as well as essentially all known $\gamma$-decaying high-spin $(J>4)$ states. This work confirms the location and the decay branching of the candidate state by Brennenstein et al. As a result, the new SD candidate band has states with $2^{+}, 4^{+}$, and $6^{+}$spinparities. Their $\gamma$-decay is strongly retarded to the oblate ground state band, and enhanced to the prolate band.

Inspired by this exciting situation with open questions on the SD state, we have carried out an independent theoretical analysis of highly-deformed structures in ${ }^{28} \mathrm{Si}$ [37]. In particular we performed a Nilsson-calculation, combined with quasi-dynamical U(3) considerations, and determined the allowed binary clusterizations of the shape isomers. As a result we have obtained 8 shape isomers from the ground state up to the alpha-chain states. The low-energy section, containing the ground (GS), prolate (Pr) and superdeformedprolate (SD) states is shown in Table 1.

Five (from our eight) shape isomers were seen previously in energy-calculations of the Nilsson model [38]. Even better agreement is recognized with Brink-model results [39-41]. These studies gave also eight shape isomers, and seven of them are in a one-to-one correspondence with our results.
Table 1. Shape isomers in the ${ }^{28} \mathrm{Si}$ nucleus from the Nilsson model + quasi-dynamical $S U(3)$ calculation. 'e' stands for effective $U(3)$ quantum numbers, ' $\mathrm{h}$ ' indicates the states corresponding to simple harmonic oscillator configurations. (p) and (o) mean prolate and oblate, respectively. The triaxiality, $\gamma$, is given in degrees. The penultimate column indicates the axis ratio, while the final column presents the moment of inertia (in units of $\hbar^{2} / \mathrm{MeV}$ ).

\begin{tabular}{|c|c|c|c|c|c|c|c|}
\hline St & Mod & $\hbar \omega$ & $\mathrm{U}(3)$ & $\beta_{2}$ & $\gamma$ & a:b:c & $\mathcal{J}$ \\
\hline \multirow{3}{*}{ GS } & $\mathrm{e}(\mathrm{p})$ & -1 & {$[13,13,9]$} & 0.17 & 60 & $1.2: 1.2: 1$ & 3.4 \\
& $\mathrm{e}(\mathrm{o})$ & 0 & {$[16,15,5]$} & 0.44 & 55 & $1.6: 1.5: 1$ & 4.8 \\
& & & & & & & 3.5 \\
& & & & & & & 3.3 \\
& $\mathrm{~h}$ & 0 & {$[16,16,4]$} & 0.50 & 60 & $1.7: 1.7: 1$ & 3.4 \\
\hline Pr & $\mathrm{e}$ & 0 & {$[19,9,8]$} & 0.44 & 5 & $1.5: 1: 1$ & 4.4 \\
& & & & & & & 4.3 \\
& & & & & & & 2.8 \\
& $\mathrm{~h}$ & 0 & {$[20,8,8]$} & 0.50 & 0 & $1.5: 1: 1$ & 4.5 \\
\hline SD & $\mathrm{e}$ & 4 & {$[27,8,5]$} & 0.81 & 7 & $2.2: 1.1: 1$ & 5.8 \\
& & & & & & & 5.5 \\
& & & & & & & 2.3 \\
& $\mathrm{~h}$ & 4 & {$[28,8,4]$} & 0.88 & 9 & $2.3: 1.2: 1$ & 6.1 \\
& & & & & & & 5.7 \\
& & & & & & & 2.2 \\
\hline \hline
\end{tabular}

We have studied systematically the allowed binary cluster configurations of the shape isomers. In doing so the clusters were considered to have deformations, like the ground states of the corresponding nuclei, and no constraint was applied for their relative orientation. The alpha-like clusterizations proved to be energetically favored.

The GS allows those clusterizations for which the lighter cluster is either ${ }^{4} \mathrm{He}$ and ${ }^{8} \mathrm{Be}$, while the $\mathrm{Pr}$ and SD states allow several cluster configurations (both the effective and the harmonic oscillator states can be clusterized). For the SD state, which was the focus of our work, we found that the ${ }^{4} \mathrm{He}+{ }^{24} \mathrm{Mg}$, and the ${ }^{12} \mathrm{C}+{ }^{16} \mathrm{O}$ cluster configurations are the most probable, taking account of both the selection rules and energetic preference. This finding is in line with the recent AMD [32] and macroscopic-microscopic calculations [33], as well as with the new experimental results [35]. Its predicted moment-of-inertia is near-identical to that of the AMD calculation, and showed by the experiment, thus it gives support to the new candidate for the SD state [35].

\section{$3.2{ }^{36} \mathrm{Ar}$}

The SD band of the ${ }^{36} \mathrm{Ar}$ nucleus was reported in [42]. Following the experimental observation a considerable theoretical effort has been concentrated on this band. In [43] e.g. the possible binary clusterizations of this state was studied systematically. Similar studies have been done also for the ground, and the hyperdeformed bands. The latter one had been predicted from alpha-cluster model calculations [44]. One of the interesting conclusions of this work [43] was that the HD state of the ${ }^{36} \mathrm{Ar}$ nucleus could be populated in the ${ }^{24} \mathrm{Mg}+{ }^{12} \mathrm{C}$ and ${ }^{20} \mathrm{Ne}+{ }^{16} \mathrm{O}$ reactions.

A recent analysis of the ${ }^{24} \mathrm{Mg}+{ }^{12} \mathrm{C}$ elastic scattering [45] revealed the fact that the cross section can be described only by supposing resonances on top of the potential scattering. This very careful analysis incorporated 


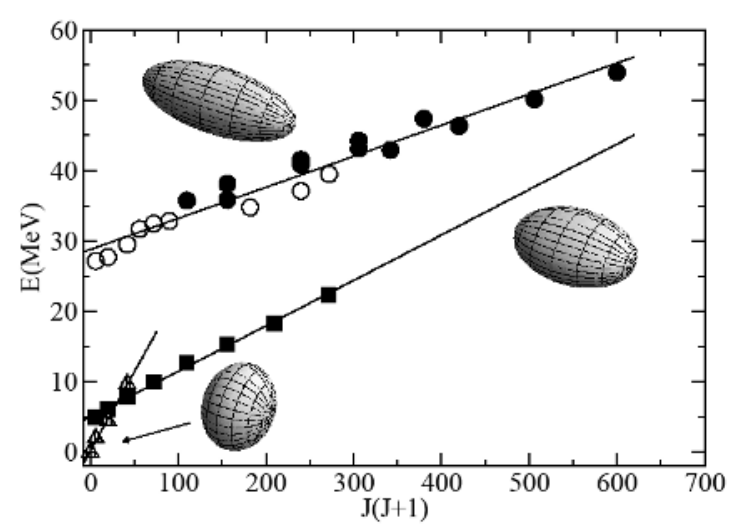

Fig. 1. Excited states in ${ }^{36} \mathrm{Ar}$, observed as resonances in the ${ }^{12} \mathrm{C}+{ }^{24} \mathrm{Mg}$ (open circles) and ${ }^{16} \mathrm{O}+{ }^{20} \mathrm{Ne}$ (full circles) reactions. The SD band observed in ${ }^{36} \mathrm{Ar}$ and the ground-state band are also included. The shapes are from Nilsson-calculations.

phase-shift study, as well as Regge-pole and energy-dependent resonance calculations. The existence of five resonances have been proved, which have angular momenta 2 , $4,6,7,8$. These states together with the resonances from the ${ }^{20} \mathrm{Ne}+{ }^{16} \mathrm{O}$ reactions seem to establish a rotational band [45], as shown in the upper part of figure 1. Its moment of inertia is in a very good agreement with that of the HD shape predicted from alpha-cluster model [44]. The similarity of the (predicted and observed) moments of inertia, and the fact that the resonances were seen in exactly those reactions, which define the preferred cluster-configurations of the HD shape suggest that the recently observed band in figure 1 is a good candidate for the hyperdeformed shape isomer of the ${ }^{36} \mathrm{Ar}$ nucleus. For comparison also the ground and superdeformed bands are indicated in figure 1. The energetical preferences of cluster-configurations have been discussed in detail in [46].

Since a candidate for the HD state showed up, the exciting question arises if such a shape can be seen in shellmodel calculation as well. In [17] we have carried out Nilsson-model+quasi-dynamical $S U(3)$ calculation in order to find the answer. This calculation gives a HD state which has exactly the same symmetry as that from the alphacluster model. The moment of inertia from these model agrees well with the one indicated by the experiment.

It was an interesting finding that different states can be built up from the same two clusters, like e.g. ${ }^{12} \mathrm{C}+{ }^{24} \mathrm{Mg}$, as illustrated in figure 2. The difference in these cases is the relative orientation of the two deformed clusters. This result is a consequence of the fact that the Pauli-principle was taken into account, and no simplifying assumptions were made on the shapes and relative orientations of the clusters.

The combination of these findings seems to suggest that the largely deformed band in figure 1 is a good candidate for the hyperdeformed state, thus ${ }^{36} \mathrm{Ar}$ may be a nucleus to have experimental evidence for the existence of ground, superdeformed, as well as hyperdeformed bands.

The question of the ternary clusterization for these shape isomers have been addressed in [47]. In particular, those possible ternary cluster-configurations have been determin-

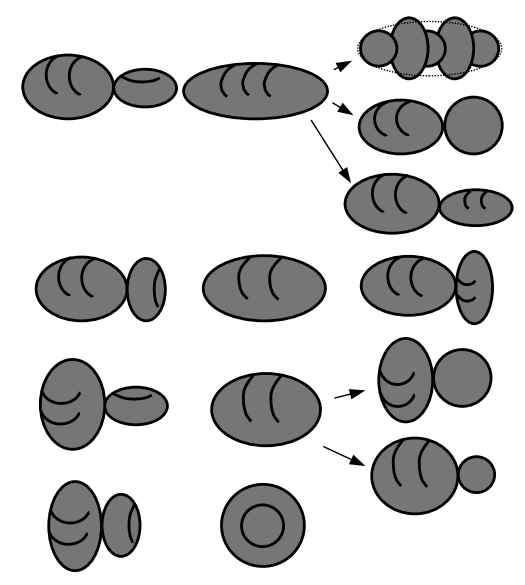

Fig. 2. The shape isomers of the ${ }^{36} \mathrm{Ar}$ nucleus from Nilssonmodel calculations, and their amalgamation from two clusters. The central part shows the shell-model results for the ground (at the bottom), superdeformed, triaxial, and hyperdeformed (at the top) states. The left column corresponds to the ${ }^{24} \mathrm{Mg}+{ }^{12} \mathrm{C}$ clusterization. The right side illustrates the ${ }^{32} \mathrm{~S}+{ }^{4} \mathrm{He},{ }^{20} \mathrm{Ne}+{ }^{16} \mathrm{O}$, ${ }^{28} \mathrm{Si}$ (prolate) $+{ }^{8} \mathrm{Be},{ }^{28} \mathrm{Si}$ (prolate) $+{ }^{8} \mathrm{Be},{ }^{20} \mathrm{Ne}+{ }^{16} \mathrm{O}$ configurations (from the bottom), respectively. At the upper most line also the alpha-clusterization of the HD state is shown from the work [44], where the contour corresponds to the HD state of the Nilssonmodel.

ed, which contain at least one double-magic clusters. They are preferred from the energetic viewpoint.

\section{$3.3^{40} \mathrm{Ca}$}

The situation of the shape isomers of the ${ }^{40} \mathrm{Ca}$ nucleus is somewhat similar to that of the ${ }^{36} \mathrm{Ar}$. In particular the SD band has been observed experimentally [48], and due to this fact several theoretical studies have been carried out. In [49] we have investigated the possible binary clusterizations in the ground, superdeformed and a hypothetical hyperdeformed band. The latter one was obtained from simple harmonic oscillator shell model configurations with quadrupole deformation, which is considered to be appropriate for the HD state. The $U(3)$ selection rule has been applied, and the energetic calculations were carried out.

Both for the SD and for the HD states the ${ }^{28} \mathrm{Si}+{ }^{12} \mathrm{C}$ clusterization turned out to be an important one. For the $\mathrm{SD}$ shape this finding is consistent with the conclusion of the fully microscopic AMD calculations [50]. Work is in progress [51] in order to complete this analysis with the systematic determination of the shape isomers from Nilsson-model+qusidynamical $U(3)$-symmetry calculations. A comparison with the results of an experimental work on the elastic scattering of ${ }^{28} \mathrm{Si}+{ }^{12} \mathrm{C}$ is also being done.

\section{$3.4^{56} \mathrm{Ni}$}

The stable elongated shapes of the ${ }^{56} \mathrm{Ni}$ nucleus, obtained from a Nilsson-model + quasi-dynamical $U(3)$ symmetry calculation, is shown in figure 3. In this figure it is not the minima, rather the horizontal plateaus, which correspond to the stable shapes. (They are insensitive to the small changes of the input parameter. Furthermore, these 


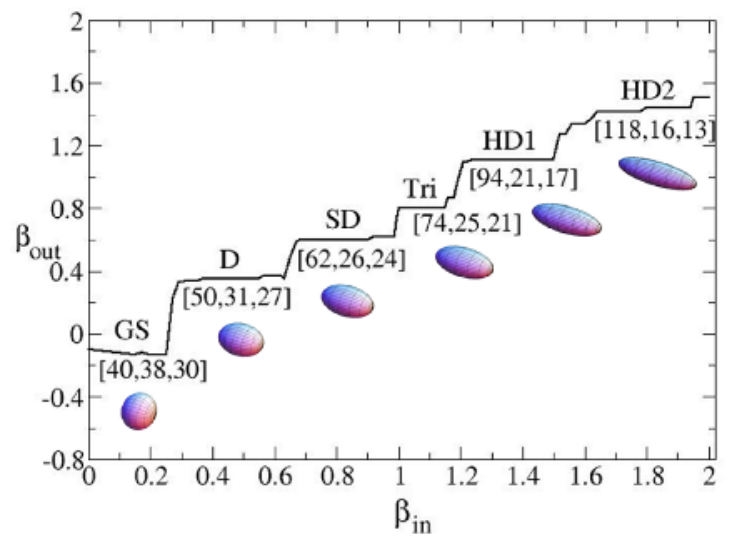

Fig. 3. Quadrupole deformation of the ${ }^{56} \mathrm{Ni}$ nucleus from the Nilsson-model with the effective $U(3)$ quantum numbers and schematic illustrations of the shape at the plateaus.

deformations fulfill the self-consistency argument between the input and output deformation-parameters to some approximation.)

As seen from the figure, the triaxial ground-state (for which the experimental deformation is $\beta_{2}=0.173$ ) is followed by a prolate-like deformed state of $0 \hbar \omega$ excitation. The next region of stability corresponds to the superdeformed shape. This state represents 4 nucleon excitation, being very much in line with $[52,53]$. Then appears an even more deformed state with triaxial shape, and two pronounced hyperdeformed shapes close to each other.

It is remarkable that a superdeformed, a triaxial and a hyperdeformed state appear both in the alpha-clustermodel calculation [53], and in our (Nilsson-model-based) quasi-dynamical symmetry consideration. The superdeformed states seem to correspond to each other exactly, both of them being a $4 \hbar \omega$ excitation. Then we observe a largely deformed triaxial state with $12 \hbar \omega$, which is not completely identical, but similar to that of the alpha-cluster model (with $16 \hbar \omega)$. This latter state is considered to be a candidate for the ${ }^{28} \mathrm{Si}+{ }^{28} \mathrm{Si}$ molecular resonances, in which the two oblate ${ }^{28} \mathrm{Si}$ are thought to have an equator-to-equator position. The alpha-cluster-model gives also a hyperdeformed state, and our calculation have two candidates for that. Based on their possible cluster-structure the lower-lying one seems to be very similar to that of the work [53].

We have found that the ground state of ${ }^{56} \mathrm{Ni}$ prefers asymmetric cluster configurations, from among the alphalike clusterization only ${ }^{4} \mathrm{He}+{ }^{52} \mathrm{Fe}$ is allowed. The deformed, superdeformed and largely deformed triaxial states match with several clusterizations. Structure considerations suggest that the correlated ${ }^{28} \mathrm{Si}+{ }^{28} \mathrm{Si}$ and ${ }^{40} \mathrm{Ca}+{ }^{16} \mathrm{O}$ resonances correspond to the superdeformed state of ${ }^{56} \mathrm{Ni}$, but not to the hyperdeformed one. In the latter case the ${ }^{40} \mathrm{Ca}+{ }^{16} \mathrm{O}$ configuration has a strong structural forbiddenness [28]. The ${ }^{24} \mathrm{Mg}+{ }^{32} \mathrm{~S}$ cluster configuration on the other hand, which is determined by the entrance channel of the ternary fission experiment [54] matches both with the SD and HD states, and with the largely deformed triaxial state in between.

The triaxial state is of special interest, because it is thought to be related to the molecular resonances of two ground-state-like (oblate) ${ }^{28} \mathrm{Si}$ clusters in their equator-toequator configuration. This configuration is allowed in the triaxial state from all cited studies. If the equator-to-equator configuration is not exactly parallel, then other alpha-like binary clusterizations, like e.g. ${ }^{24} \mathrm{Mg}+{ }^{32} \mathrm{~S}$, are also possible.

The hyperdeformed state both from the alpha-cluster and from our Nilsson-calculation prefers a binary configuration of prolate ${ }^{28} \mathrm{Si}$ clusters with a pole-to-pole configuration. The state from our quasi-dynamical considerations allows ${ }^{24} \mathrm{Mg}+{ }^{32} \mathrm{~S}$ as well (again close to the position in which the longest major axes of both nuclei are parallel with the molecular axis). The HD state from alpha-cluster studies does not contain this configuration.

The energetic preference of the cluster configurations were obtained from binding-energy arguments [25], and from calculations based on the Dinuclear System Model [26]. The latter ones were performed both for the poleto-pole configurations and for the ones derived from the microscopic considerations. The ${ }^{4} \mathrm{He}+$ core configuration turned out to be the most preferred one, followed by the ${ }^{8} \mathrm{Be}+$ core one. Then a group of the ${ }^{12} \mathrm{C},{ }^{28} \mathrm{Si}$, and ${ }^{16} \mathrm{O}$ clusters come, with close-lying values, but in different order from different calculations. The ${ }^{24} \mathrm{Mg}$ and ${ }^{20} \mathrm{Ne}$ turned out to be the least-preferred alpha-like clusters.

More detailed investigations on this nucleus is presented in [28]. In this work the results of the energetic calculation with three different methods are compared: in addition to the binding energy, and double folding, mentioned above, there the extended liquid drop model [55] was also applied.

\subsection{Other $\mathbf{N}=\mathbf{Z}$ nuclei}

A comparison of the possible (predicted) alpha-like binary cluster configurations in the SD and HD states of these nuclei, as well as of the the ${ }^{60} \mathrm{Zn}$ nucleus is presented in [56]. That work includes also further discussion on the candidate reactions to populate these shape isomers.

\section{Summary}

In this paper we have considered the elongated shape isomers of some $N=Z$ nuclei and their possible binary clusterizations. Both in finding the stable shapes and in determining their relations to cluster configurations symmetry considerations were applied extensively. It could be done due to the facts that i) the $U(3)$ symmetry turned out to recover for these states even for realistic (Nilsson-type) interactions, and ii) the number of shell-model configurations which build up the cluster state reduce considerably.

We have determined the shape isomers from the quasidynamical $U(3)$ symmetry, obtained from Nilsson-calculations.

In searching for the possible binary clusterizations of the shape isomers we have taken into account both natural laws which govern the building up of a nucleus from smaller constituents. The exclusion principle was taken into account by applying a selection rule (in combination with Harvey's prescription), based on the microscopic configuration associated to the quasi-dynamical $U(3)$ symmetry. In this way the Pauli-principle is incorporated only in an approximate way, of course. But it is done in a welldefined procedure, which can be checked in simple systems by comparing with exact results. This approximation 
can be illustrated in simple geometrical terms, in spite of its abstract algebraic content: it measures, how similar the quadrupole deformations are in the cluster configuration and in the shell-model (or collective model) state.

The clusters were considered to have a deformation, like the corresponding free nuclei (spherical, prolate, oblate or triaxial), and no constraints were applied for their relative orientation.

The methods we applied here seem to be applicable in heavier nuclei, too. Symmetry considerations can be helpful in studying both the shape isomers of nuclei, and their clusterizations. As for this latter problem is concerned we think that the preferred cluster configurations are those ones which are favored by the energetics, and which are Pauliallowed. From the viewpoint of the application to heavier systems we consider it as a promising sign, that the results of the present method [49] are very similar to the ones from $\mathrm{ab}$ initio calculations for the case of the ${ }^{40} \mathrm{Ca}$ nucleus [50], where the fully microscopic treatment was also applied.

\section{Acknowledgment}

This work was supported by the OTKA (Grant No K72357), TÁMOP (4.2.1./B-09/1/KONV-2010-0007/IK/IT), and MTAJINR collaboration (project no 2009/001).

\section{References}

1. J.P. Elliot, Proc. Roy. Soc. A 245, 128 (1958)

2. K. Wildermuth and Th. Kanellopoulos, Nucl. Phys. 7, 150 (1958)

3. B.F. Bayman and A. Bohr Nucl. Phys. 9, 596 $(1958 / 59)$

4. G. Rosensteel and J.P. Draayer, J. Phys. A 22, 1323 (1989)

5. K. Sugawara-Tanabe and A. Arima, Nucl. Phys. A 619, 88 (1997)

6. K. Sugawara-Tanabe, K. Tanabe, A. Arima, and B. Gruber, Phys. Rev. C 80, 044307 (2009)

7. W.D.M. Rae, Int. J. Mod. Phys. 3, 1343 (1988)

8. W. Nazarewitch and J. Dobaczewski, Phys. Rev. Lett. 68, 154 (1992)

9. J. Cseh and W. Scheid, J. Phys. G: Nucl. Part. Phys. 18, 1419 (1992)

10. K. Sugawara-Tanabe and A. Arima, Phys. Lett. B 317, 1 (1993)

11. K. Sugawara-Tanabe, A. Arima, and N. Yoshida, Phys. Rev. C 51, 1809 (1995)

12. P. Rochford and D. J. Rowe, Phys. Lett. B 210, 5 (1988);

D. J. Rowe, P. Rochford, and J. Repka, J. Math. Phys. 29, 572 (1988)

13. J. Cseh, in: Proceedings of IV Intl. Symposium on Quantum Theory and Symmetries (Varna, Bulgaria) (Sofia, Heron Press) (2006) p. 918.

14. M. Jarrio, J. L. Wood, and D. J. Rowe, Nucl. Phys. A 528, 409 (1991)

15. P. O. Hess, A. Algora, M. Hunyadi, and J. Cseh, Eur. Phys. J. A 15, 449 (2002)

16. D. J. Rowe, Rep. Prog. Phys. 48, 1419 (1985)
17. J. Cseh, J. Darai, W. Sciani, Y. Otani, A. Lepine-Szily, E.A. Benjamim, L.C. Chamon, and R. Lichtenthaler, Phys. Rev. C 80, 034320 (2009)

18. J. Cseh, J. Darai, A. Algora, H. Yepez-Martinez, and P. O. Hess, Rev. Mex. Fis. S. 54 (3), 0030 (2008)

19. J. Cseh and J. Darai, AIP Conf. Proc. 1098: Fusion08 225 (2008), and references therein.

20. J. Cseh and J. Darai, Acta Univ. Debrecen., Ser. Phys. Chim. XL, 23 (2006)

21. K.T. Hecht, Phys. Rev. C 16, 2401 (1977)

22. Y. Suzuki, Nucl. Phys. A 448, 395 (1986)

23. M. Harvey, in: Proceedings of 2nd Intl. Conference on Clustering Phenomena in Nuclei, (College Park); USDERA report ORO-4856-26, p. 549 (1975)

24. A. Algora and J. Cseh, J. Phys. G: Nucl. Part. Phys. 22, L39 (1996)

25. B. Buck, A. C. Merchant, and S. M. Perez, Few-Body Systems 29, 53 (2000)

26. V.V. Volkov, Phys. Rep. 44, 93 (1978); Deep inelastic nuclear reactions (Energoizdat, Moscow, 1982)

27. T. M. Shneidman, et al., Phys. Lett. B 526, 322 (2002)

28. J. Darai, J. Cseh, N.V. Antonenko, G. Royer, A. Algora, P.O. Hess, R.V. Jolos, and W. Scheid, Phys. Rev. C 84, 024302 (2011)

29. G. Audi, A. H. Wapstra, and C. Thibault, Nucl. Phys. A 729, 337 (2003)

30. S. Raman, C. W. Nester, and P. Tikkanen, At. Data Nucl. Data Tables 78, 1 (2001)

31. G.G. Adamian, N.V. Antonenko, S.P. Ivanova, R.V. Jolos, and O. I. Melnikova, Int. J. Mod. Phys. E 5, 191 (1996)

32. Y. Taniguchi, Y. Kanada-En'yo, and M. Kimura, Phys. Rev. C 80, 044316 (2009)

33. T. Ichikawa, Y. Kanada-En'yo, and P. Moller, Phys. Rev. C 83, 054319 (2011)

34. S. Kubono, et al., Nucl. Phys. A 457, 461 (1986)

35. D.G. Jenkins, et al. submitted to Phys. Rev. C.

36. J. Brenneisen, et al., Z. Phys. A 352, 149 (1995); ibid. p.279; ibid. p.403.

37. J. Darai, J. Cseh, and D.G. Jenkins, submitted to Phys. Rev. C.

38. G. Leander and S.E. Larsson, Nucl. Phys. A 339, 93 (1975)

39. A.C. Merchant and W.D.M. Rae, Nucl. Phys. A 549, 431 (1992)

40. J. Zhang and W.D.M. Rae, Nucl. Phys. A 564, 252 (1993)

41. J. Zhang, W.D.M. Rae, and A.C. Merchant, Nucl. Phys. A 575, 61 (1994)

42. C.E. Svensson, et al., Phys. Rev. Lett. 85, 2693 (2000)

43. J. Cseh, A. Algora, J. Darai, and P.O. Hess, Phys. Rev. C 70, 034311 (2004)

44. W.D.M. Rae and A.C. Merchant, Phys. Lett. B 279, 207 (1992)

45. W. Sciani, Y. Otani, A. Lepine-Szily, E.A. Benjamim, L.C. Chamon, R. Lichtenthaler, J. Darai, and J. Cseh, Phys. Rev. C 80, 034319 (2009)

46. J. Cseh, J. Darai, N.V. Antonenko, G.G. Adamian, A. Algora, P.O. Hess, and A. Lepine-Szily, J. Phys. Conf. Ser. 239, 012006 (2010)

47. A. Algora, J. Cseh, J. Darai, and P.O. Hess, Phys. Lett. B 639, 451 (2006) 


\section{NSRT12}

48. E. Ideguchi, et al., Phys. Rev. Lett. 87, 222501 (2001); S. Torilov, et al., Eur. Phys. J. A 19, 307 (2004)

49. J. Cseh, J. Darai, N.V. Antonenko, A. Algora, P.O. Hess, R.V. Jolos, and W. Scheid, Rev. Mex. Fis. S. 52 (4), 11 (2006)

50. Y. Kanada-Enyo, M. Kimura, and H. Horiuchi, AIP Conf. Proc. 644, 188 (2003)

51. J. Darai, A. Lepine-Szily et al., in preparation.

52. D. Rudolph, et al., Phys. Rev. Lett. 82, 3763 (1999)

53. J. Zhang, A. C. Merchant, and W. D. M. Rae, Phys. Rev. C 49, 562 (1994)

54. W. von Oertzen, et al., Eur. Phys. J. A 36, 279 (2008)

55. G. Royer and B. Remaud, Nucl. Phys. A 444, 477 (1985)

56. J. Cseh, J. Darai, A. Algora, N.V. Antonenko, and G.G. Adamian, EPJ Web of Conf. 17, 16001 (2011) 